\title{
CMS Patatrack project
}

A. Bocci ${ }^{1}$, V. Innocente ${ }^{1}$, M. Kortelainen ${ }^{2}$, F. Pantaleo $^{1}$, M. Rovere ${ }^{1}$ $C E R N^{1}, F N A L^{2}$

\section{Joint HSF/OSG/WLCG Workshop}

March 19, 2019 


\section{The Patatrack group}

- Patatrack was formed by people with common interest and a varied pool of expertise

- Software optimisation

- Heterogeneous architectures

- Track reconstruction

- High Level Trigger

- Work started in 2016 with the participation to the EuroHack 2016 event, sponsored by NVIDIA

- And continued through 2017 to 2019 with self-organized Hackathons at CERN, collaboration with Openlab, training and working with students, and so on 


\section{The Patatrack demonstrator}

- Goal is demonstrate that part of the HLT reconstruction can be efficienty offloaded

- Running on a single machine equipped with GPUs

- Focus on a $\sim 10 \%$ slice of HLT time consumption

- Pixel local reconstruction

- Pixel-only track reconstruction

- Vertex reconstruction

- Other groups have started to work on

- Calorimeters local reconstruction

- Full track reconstruction

- For more details see closeby talks in

- ACAT 2019, 10-15 March, Saas-Fee (Switzerland)

- CDT/WIT 2019, 2-5 April, Valencia (Spain) 


\section{The Patatrack demonstrator workflow}

- Copy the pixel raw data to the GPU

- Pixel local reconstruction

- Decode the raw data

- Clustering

- Calibrations

- Pixel-only tracking

- Form hit doublets

- Form hit quadruplets with Cellular automaton algorithm

- Optionally

- Full track fit (Riemann, Broken-line fits)

- Some GPU algorithms are same, others different wrt. (legacy) CPU

- Implementations are currently different

- Bitwise or statistically identical physics performance

- Organized as a chain of 3 GPU producer modules

- Pass GPU data from one producer to the next

- Use the CMSSW's "external worker" mechanism 


\section{The Patatrack demonstrator (2018)}

CMS Preliminary 2018 data $13 \mathrm{TeV}$

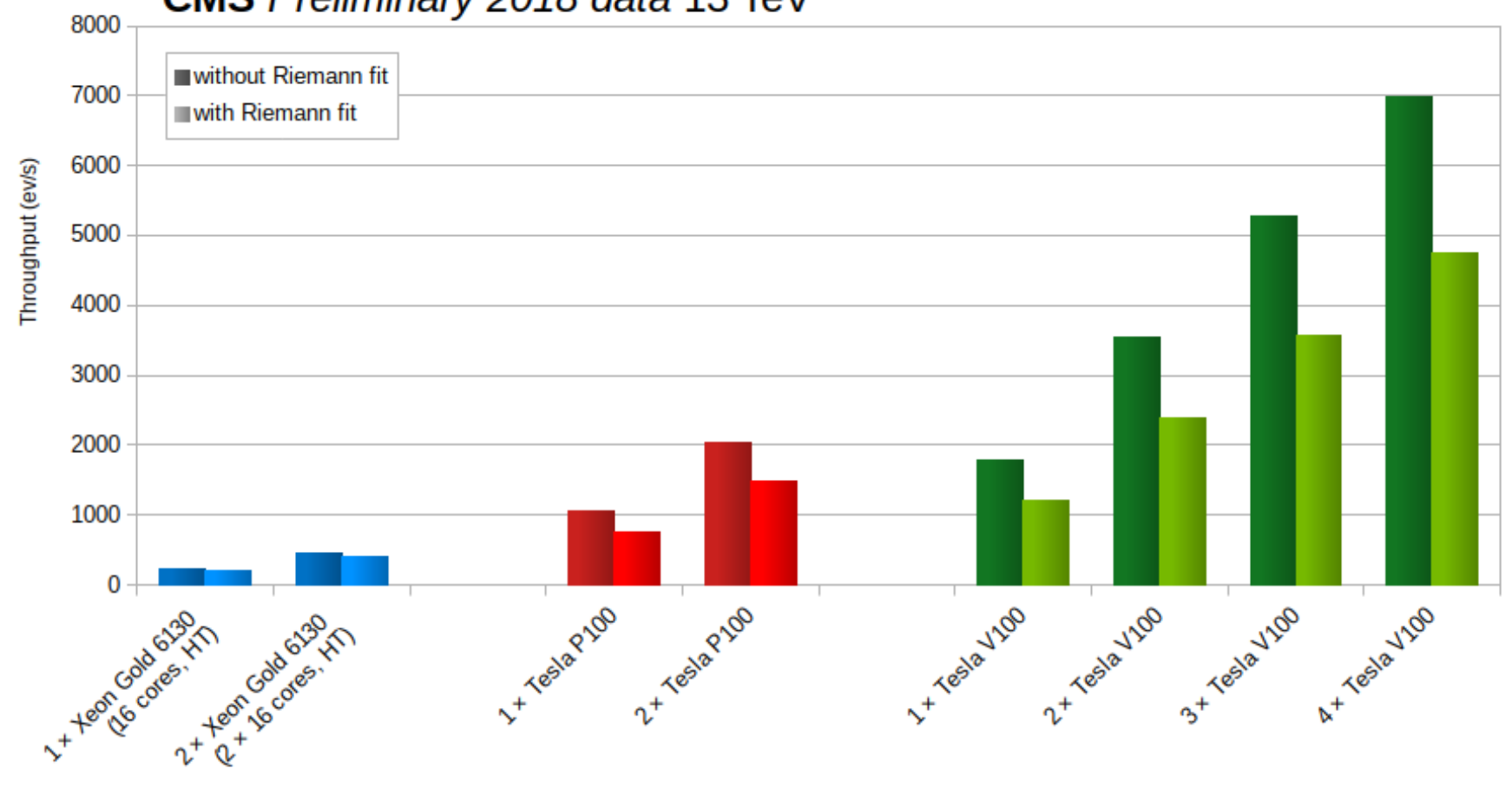

- 2018 data: average pileup 50

- HLT-like configuration, optimised for maximal throughput

- One Tesla V100 is 5×-7× faster than one Xeon Gold 6130 


\section{CPU utilization}

modules running event stalled module running

modules running other multiple modules running
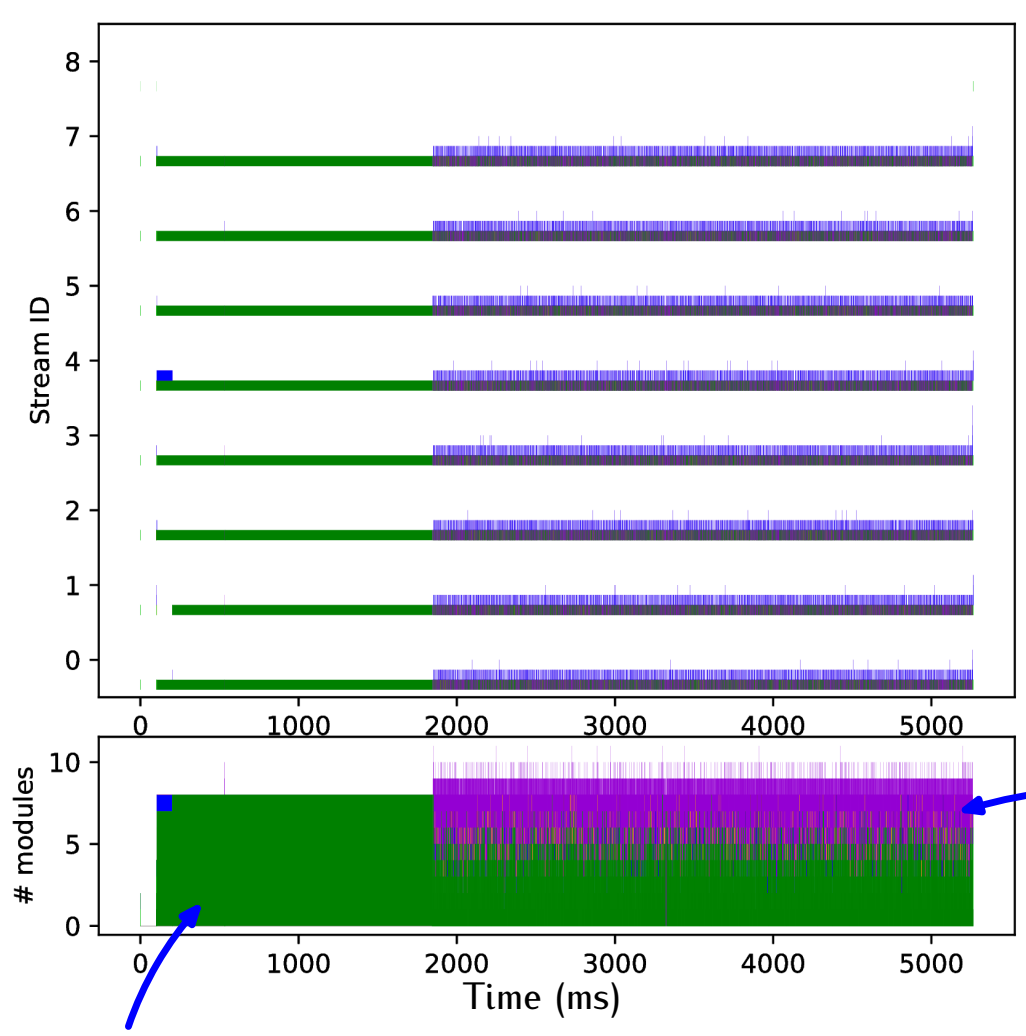

Number of running modules read from input external work

- Caveat: different machine (i7-4771, GeForce RTX 2080)

-8 threads and 8 concurrent events

- After the initialization

- CPU utilization is roughly $50 \%$

- There are roughly 4-5 external workers scheduled in parallel

- NB: this workflow is "artificially" tuned to minimize the $\mathrm{CPU}$ utilization

Number of scheduled external workers 


\section{GPU utilization}
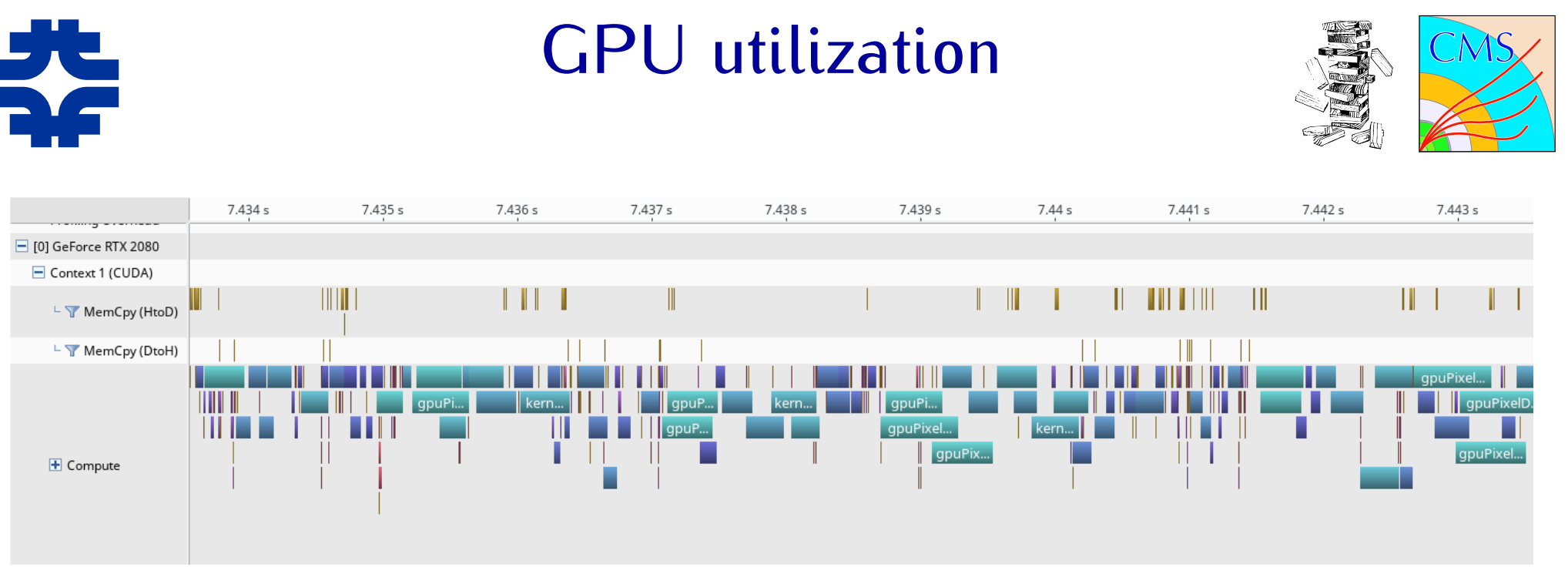

- Screenshot of NVIDIA Visual Profiler for a random $10 \mathrm{~ms}$ period

- Kernels and data transfers being run in parallel 


\section{Lessons learned: design principles}

- For optimal performance, follow a Data Oriented Design

- Memory operations are costly, computations are almost free

- Design the data structure for maximal efficiency (SOA vs ... vs. AOS)

- Implement the algorithms around the data structure

- Avoid object-oriented patterns in critical code e.g. data formats $\star$ inheritance, virtual functions, etc

- Most (all?) GPU operations (memory copies, running "kernels", etc) should be asynchronous

- The "kernels" run on the GPU while the CPU is doing other work

- The GPU can transfer data to and from the host while both the CPU and the GPU are working

- Memory transfer, and especially data format conversions, between CPU and GPUs are costly

- In some cases, almost as much as running the original algorithm itself 


\section{Lessons learned: tools and architectures}

- CUDA and CMSSW support different sets of compilers and C++ features

- CUDA 10.1 supports

$$
\star \mathrm{C}++14
$$

$\star$ GCC 8, CLANG 7

$\triangle$ CUDA 10.0 supported GCC 7, CLANG 6

- CMSSW 10.6.X supports

$\star \mathrm{C}++17$

$\star$ GCC 7 and GCC 8, CLANG 7

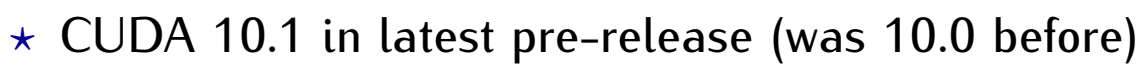

- Unfortunately, we need to keep the host and device code somewhat separate

- Host code can use $\mathrm{C}++17$ features

- Device code (and common code) is limited to $\mathrm{C}++14$ features

- You do not want to \#include framework (or ROOT) headers in device code! 


\section{Lessons learned: what about CMSSW?}

- Redesign dedicated data formats for use on GPUs

- In fact, they might be more efficient also on traditional CPUs

- Design a chain of algorithms (framework modules) that work on the GPU

- Without copying data back and forth

- Take advantage of the "external worker" approach in CMSSW

- Launch the work on the GPU, schedule other work in parallel on the CPU

- Split GPU modules in two parts

- The part that deals with the framework and the rest of the CMSSW

- The part that deals with the GPU data structures and kernels

- Split the GPU-related work in two (or more) modules, e.g.

- Copy data from CPU to GPU, launch kernels

- Copy data from GPU to CPU * ran only if another modules consumes the CPU SOA

- Transform CPU SOA to CPU legacy data format $\star$ ran only if another module consumes 


\section{Model for CUDA Producers}

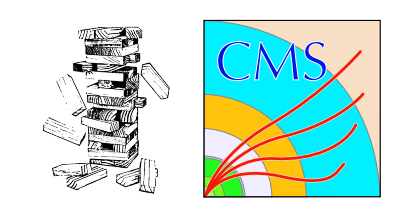

- Aim to avoid blocking synchronization as much as possible

- A helper object gives the CUDA device and stream to use for the algorithms

- Memory management

- Raw CUDA allocations and frees should be avoided within the event loop

- Preallocating memory buffers as module member data leads to unnecessarily high GPU memory use

- We went for a caching allocator for device and pinned-host memory that amortizes the cost of raw CUDA allocations

$\star$ Currently based on the caching allocator of cub

- GPU event products are like regular EDM products, but enclosed in a wrapper that holds also the CUDA device and the CUDA stream

- Allows the consumer to set the device, and queue more work to the same CUDA stream

- Allows also the TBB-flowgraph streaming_node style operation * Module in the middle of the chain may only queue more asynchronous work $\star$ Later module in the chain synchronizes (with "external worker") 


\section{Conclusions}

- We have demonstrated that GPUs are an efficient alternative to traditional CPUs

- For complex tasks like track reconstruction

- Next steps

- Integrate the developments in the official CMSSW

- Continue evolving the framework to make it easier to leverage GPUs

- Focus on code portability and avoiding code duplication as much as possible

- Study how more algorithms and data structures could benefit from GPUs

- Study local vs. remote offloading to GPUs 


\section{BACKUP MATERIAL}




\section{The Patatrack demonstrator (2018)}
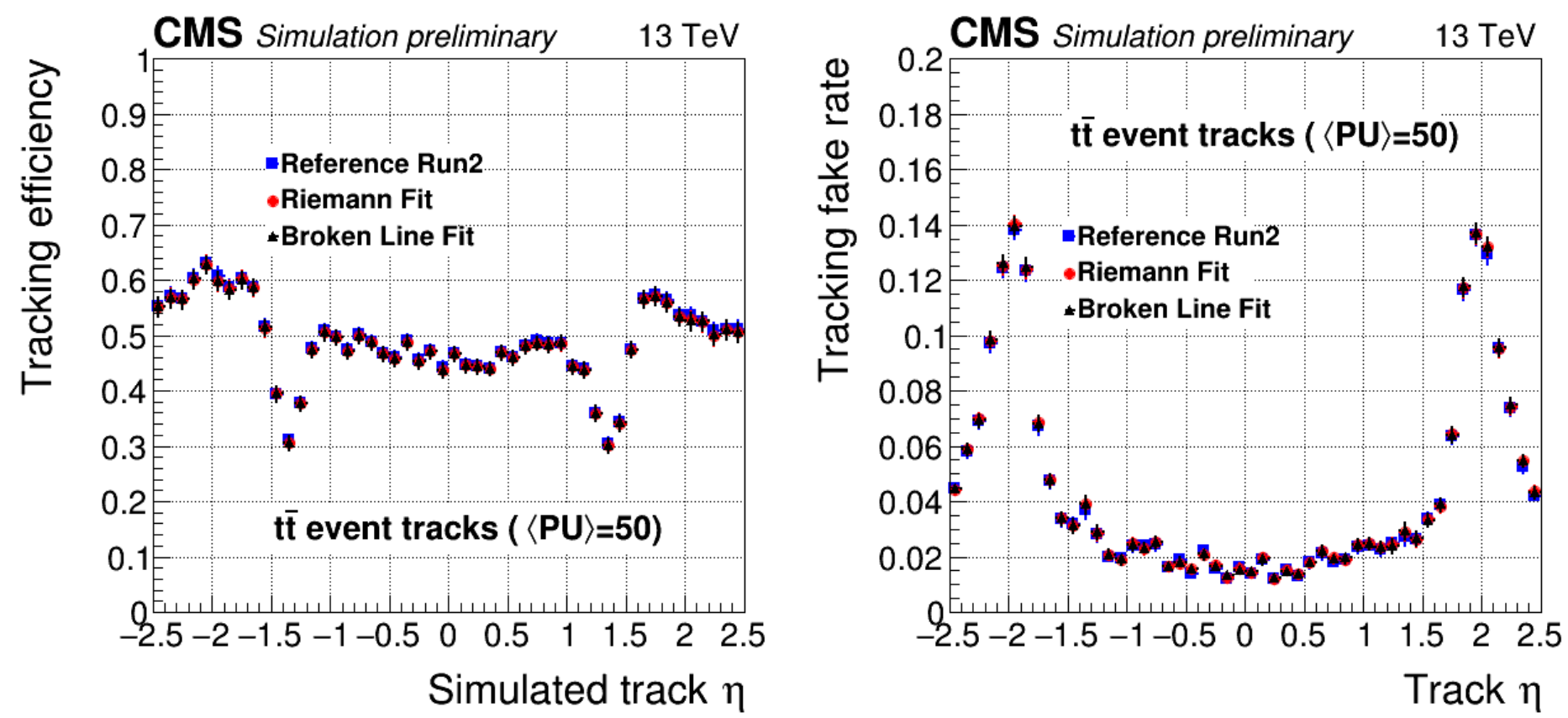

- Similar efficiency and fake rate as with legacy CPU algorithm

- More information: CMS Detector Performance Note DP-2018/059 


\section{The Patatrack demonstrator (2018)}
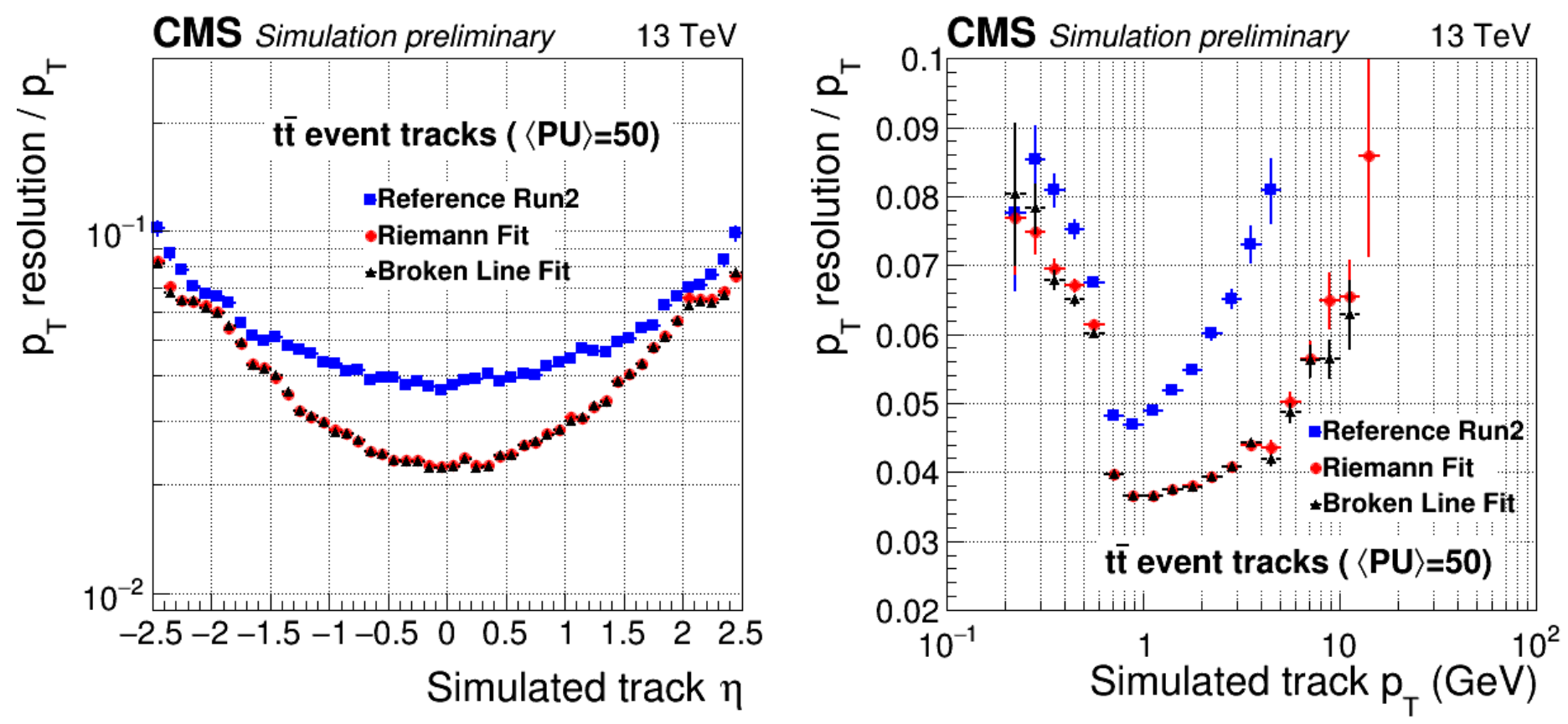

- Proper fits improve resolution significantly

- More information: CMS Detector Performance Note DP-2018/059 